OPEN ACCESS

Edited by:

Gregor Thut,

University of Glasgow,

United Kingdom

Reviewed by:

Christopher Gundlach,

Leipzig University, Germany

Danielle D. Sliva,

Brown University, United States

*Correspondence:

Hirotake Yokota

yokota@nuhw.ac.jp

Specialty section:

This article was submitted to

Perception Science,

a section of the journal

Frontiers in Neuroscience

Received: 26 June 2020 Accepted: 20 January 2021

Published: 18 February 2021

Citation:

Yokota H, Otsuru N, Saito K, Kojima S, Miyaguchi S, Inukai Y, Nagasaka K and Onishi H (2021)

Region-Specific Effects of $10-\mathrm{Hz}$

Transcranial Alternate Current

Stimulation Over the Left Posterior

Parietal Cortex and Primary

Somatosensory Area on Tactile

Two-Point Discrimination Threshold.

Front. Neurosci. 15:576526.

doi: 10.3389/fnins.2021.576526

\section{Region-Specific Effects of $10-\mathrm{Hz}$ Transcranial Alternate Current Stimulation Over the Left Posterior Parietal Cortex and Primary Somatosensory Area on Tactile Two-Point Discrimination Threshold}

\author{
Hirotake Yokota ${ }^{1,2 *}$, Naofumi Otsuru1,2, Kei Saito 1,2, Sho Kojima ${ }^{1,2}$, Shota Miyaguchi1,2, \\ Yasuto Inukai ${ }^{1,2}$, Kazuaki Nagasaka ${ }^{1,2}$ and Hideaki Onishi1,2 \\ ${ }^{1}$ Institute for Human Movement and Medical Sciences, Niigata University of Health and Welfare, Niigata, Japan, \\ ${ }^{2}$ Department of Physical Therapy, Niigata University of Health and Welfare, Niigata, Japan
}

Changes in $\alpha$-band cortical oscillatory activity $(8-13 \mathrm{~Hz})$ affect perception; however, how these changes in the left posterior parietal cortex (PPC) and primary somatosensory cortex (S1), which play different roles in determining the two-point discrimination (TPD) threshold, affect TPD threshold remains unelucidated. Therefore, to determine TPD threshold, we aimed to investigate the function of the left PPC and S1 by applying $\alpha$-band transcranial alternating current stimulation ( $\alpha$-tACS; $10 \mathrm{~Hz}$ ). TPD threshold was examined at the pad of the right index finger, contralateral to the stimulation site, in 17 healthy adults using a custom-made, computer-controlled, two-point tactile stimulation device, with random application of either active or sham $\alpha$-tACS over the left PPC (Experiment 1) and left S1 (Experiment 2). Then, 50\% TPD threshold was obtained in the active and sham conditions via logistic regression analysis. Afterward, we compared the difference between the active and sham conditions at 50\% TPD threshold in each region and found that $\alpha$-tACS reduced TPD threshold when applied over the left PPC ( $P=0.010)$; however, its effect was insignificant when applied over the left S1 $(P=0.74)$. Moreover, a comparison of the change in 50\% TPD threshold among the regions revealed that $\alpha$-tACS applied over the left PPC significantly reduced TPD threshold compared with that applied over the left $S 1(P=0.003)$. Although we did not reveal the actual changes in cortical activity induced by $\alpha$-tACS, this is the first empirical evidence that $\alpha$-tACS applied over the left PPC and left S1 exerts regionspecific effects on determining TPD threshold assessed in the contralateral index finger pad by stimulation.

Keywords: transcranial alternating current stimulation, $\alpha$-band activity, two-point discrimination, posterior parietal cortex, primary somatosensory cortex 


\section{INTRODUCTION}

Haptic information from the outside world is input to areas $3 \mathrm{~b}$ and 1 of the primary somatosensory cortex (S1) by ascending afferent fibers, such as the slowly adapting fibers and rapidly adapting fibers, and is then sent to the secondary somatosensory cortex (S2) and higher-order areas, such as the posterior parietal cortex (PPC) (Inui et al., 2004). Two-point discrimination (TPD), a type of tactile function, has been extensively used in neurophysiological research and clinical practice since it was proposed in 1834 by Weber as a measurement of higher-order perceptual functions. Moreover, TPD threshold increases with aging and certain clinical conditions, such as central or peripheral nerve involvement and non-specific low back pain (Bassetti et al., 1993; Stevens and Cruz, 1996; Heriseanu et al., 2005; Catley et al., 2014a; Harvie et al., 2018). Conversely, it has been reported that TPD threshold decreases by tactile or electrical stimulation to the measurement site (Godde et al., 2000; Ragert et al., 2003, 2008; Tegenthoff et al., 2005; Dinse et al., 2006) and that improvements in TPD positively correlate with the enlarged area of the blood-oxygen-level-dependent signal (Pleger et al., 2003) and gray matter volume in the S1 (Schmidt-Wilcke et al., 2018). Furthermore, the inferior parietal lobule within the left PPC, a major region involved in attentional control (Corbetta et al., 2000; Yantis et al., 2002), is reportedly activated when performing a TPD task rather than during a simple sensory detection task (Sripati et al., 2006; Akatsuka et al., 2007, 2008; Pleger et al., 2016). Therefore, both S1 and PPC play important roles in the TPD process.

Furthermore, alpha $(\alpha)$-band oscillatory activity $(8-13 \mathrm{~Hz})$ in the cortex reportedly affects the process of sensory perception in the brain. Perceptual performance negatively correlates with the power of $\alpha$-band activity in the visual (Van Dijk et al., 2008; Iemi et al., 2017) and somatosensory (Haegens et al., 2011b; Lange et al., 2012; Baumgarten et al., 2016; Craddock et al., 2017) domains. The underlying mechanisms of these phenomena comprise $\alpha$-band activity exerting a gating effect that suppresses signal transduction by increasing its power in areas that are not involved in the task at hand (Jensen and Mazaheri, 2010; Foxe and Snyder, 2011; Jensen et al., 2014; Van Diepen et al., 2019). This modulation of $\alpha$-band activity is considered to be controlled by top-down attention modulation in both the visual (Thut et al., 2006; Gould et al., 2011; Jensen et al., 2014) and the somatosensory (Jones et al., 2010; Zhang and Ding, 2010; Haegens et al., 2011a) domains. Therefore, in the somatosensory cortex, $\alpha$-band activity is considered to have an inhibitory role.

Conversely, increased $\alpha$-band activity is reportedly associated with improved performance requiring sustained attention, such as in a working-memory task, where higher-order functions are involved (Makeig and Inlow, 1993; Dockree et al., 2007; Braboszcz and Delorme, 2011). For example, Dockree et al. (2007) reported that a higher $\alpha$-band power predicts good sustained attention performance and that the oscillatory $\alpha$-band activity correlated to it is generated in the parietal and occipital lobes. That is, while the $\alpha$-band activity in the $S 1$ has an inhibitory role, it may have a facilitative role in the PPC, which is involved in attentional control.
However, recent studies have suggested that modulation of $\alpha$-band activity affects decision confidence and awareness of sensory experiences rather than sensitivity during discrimination tasks in the visual domain (Samaha et al., 2017, 2020; Iemi and Busch, 2018). In addition, as previously mentioned, the role of $\alpha$-band activity varied among the studies because it plays different roles depending on the requirements during information processing. Although the S1 and PPC are important regions for somatosensory information processing (Inui et al., 2004; Jones et al., 2009; Haegens et al., 2010; Gundlach et al., 2017) and have been proposed as generators of $\alpha$-band activity (Dockree et al., 2007; Jones et al., 2009; Halgren et al., 2019), the manner in which they affect TPD, a higherorder somatosensory perception, remains unclear. Owing to the hierarchal requirement of information processing in the S1 and PPC, the role of $\alpha$-band activity might be different depending on the brain regions; therefore, modulation of $\alpha$-band activity in these regions may have different effects on TPD performance.

Transcranial alternating current stimulation (tACS) has attracted considerable attention as a non-invasive method to modulate cortical oscillatory activity (Helfrich et al., 2014; Vossen et al., 2015). This type of stimulation can only synchronize oscillatory neural activity in the cortical area beneath the stimulus and artificially enhance cortical oscillation in that frequency range when applied over the occipital (Zaehle et al., 2010; Helfrich et al., 2014; Vossen et al., 2015) and somatosensory (Berger et al., 2018) cortices. However, the effects of $\alpha$-tACS on somatosensory perception have also been reported to remain unchanged (Sliva et al., 2018) or to decrease after stimulation (Gundlach et al., 2017); therefore, there is no unified view yet. Furthermore, a recent study of tACS on the motor system suggested that the reported effect of tACS is not dominated by transcranial stimulation to the brain but by transcutaneous stimulation of the peripheral nerves (Asamoah et al., 2019); studies on rats and cadavers have reported that high-intensity currents of $>4 \mathrm{~mA}$ are required to directly phase-entrain brain rhythms (Vöröslakos et al., 2018). Because we have not measured actual brain oscillatory activity during $\alpha$-tACS and have not conducted an electrical field simulation, there were many limitations to our design to strongly address in our initial hypothesis. Although the exact mechanism of tACS is still under investigation, a previous study reported that $\alpha$-tACS increases somatosensory $\alpha$-band activity immediately after stimulation (Berger et al., 2018). Further, another study reported that tACS may at least impact circuit dynamics (Sliva et al., 2018); therefore, we conducted the present experiments based on the hypothesis that $\alpha$-tACS may increase $\alpha$-band activity.

Considering these findings, although the change in the actual oscillatory brain activity caused by $\alpha$-tACS remains unknown in the present study, we hypothesized that $\alpha$-tACS may decrease TPD threshold when applied over the left PPC because increased $\alpha$-band activities are reported to facilitate sustained attention (Dockree et al., 2007; Clayton et al., 2018). Conversely, $\alpha$-tACS' application to the left S1 may increase TPD threshold because it inhibits information processing to the higher-order regions (Jensen and Mazaheri, 2010; Foxe and Snyder, 2011; Jensen et al., 2014; Van Diepen et al., 2019). We tested our hypothesis 
by measuring TPD threshold, a higher-order somatosensory function that requires sustained attention, after applying $10-\mathrm{Hz}$ $\alpha$-tACS to the left PPC (Experiment 1) and S1 (Experiment 2 ), wherein these regions were believed to play different roles in information processing (Corbetta et al., 2000; Yantis et al., 2002; Akatsuka et al., 2007, 2008). Furthermore, by comparing the differences in TPD thresholds in the two regions based on the results of Experiments 1 and 2, we found the cortical region that effectively improved TPD following $\alpha$-tACS of either the left PPC or S1.

\section{MATERIALS AND METHODS}

\section{Participants}

Overall, 21 healthy college students (12 men and nine women; age, $20.5 \pm 0.8$ years) with no history of neurological, orthopedic, or psychiatric disorders and who were not receiving any medication during the experimental period were included in this study. All participants underwent the experiments via the application of $\alpha$-tACS to the left PPC (Experiment 1) and left S1 (Experiment 2). In the TPD task, four participants who answered two points to a one-point stimulus were excluded from the analysis to maintain the consistency of the task; as a result, final analysis included 17 participants. This experiment was conducted in accordance with the Declaration of Helsinki and was approved by the Ethics Committee of Niigata University of Health and Welfare (18264-19092). Full written informed consent was obtained from all participants.

\section{tACS}

Transcranial alternating current stimulation was delivered using a battery-driven, constant current stimulator (Eldith, neuroConn $\mathrm{GmbH}$, Ilmenau, Germany) through a pair of saline-soaked surface sponge electrodes $\left(5 \times 5 \mathrm{~cm}, 25 \mathrm{~cm}^{2}\right)$. One of the electrodes was placed over the left PPC (P3 according to the international 10-20 method; Experiment 1) or left S1 $(2 \mathrm{~cm}$ posterior to C3; Experiment 2), whereas the other electrode was placed on the lateral side of the contralateral shoulder (Figures 1A,B). The reason for placing the reference electrode on the lateral side of the shoulder is that phosphenes reportedly occur during low-frequency tACS at low frequencies, which may affect the results of the measurements (Raco et al., 2014). Specifically, it is possible that the presence of phosphenes may alert the participants about the application of tACS or sham stimulation during the experiment; as a result, the correct answer rate may be affected by the input of sensory information unrelated to the task during the TPD task. Previous studies have solved this issue by placing a reference electrode on the lateral side of the shoulder; therefore, we adopted the same method in this experiment (Mehta et al., 2015; Miyaguchi et al., 2018). Although Mehta et al. (2015) reported that the extracephalic reference resulted in increased stimulation effects of the subcortical brain regions, it has also been reported that the reference electrode placed over the contralateral shoulder to the transcranial direct current stimulation (tDCS) for the dorsolateral prefrontal cortex (DLPFC) improves attentional bias compared with that placed over the left DLPFC (Shahbabaie et al., 2018). Moreover, the current density by tDCS remained the same regardless whether the position of the reference electrode was on the right cheek or right shoulder (Im et al., 2012). Therefore, we assumed that similar results were obtained with tACS and decided to use the extracephalic reference electrode. tACS was applied at $10 \mathrm{~Hz}$ with a sinusoidal wave with a constant current intensity of $1.0 \mathrm{~mA}$ (peak-to-peak). The impedance was maintained at $<10 \mathrm{k} \Omega$ during the stimulation in accordance with the latest tACS guidelines (Antal et al., 2017).

\section{TPD Threshold}

For TPD measurements, the participants were seated in a resting position on a chair with a backrest and the right shoulder and elbow joints were placed in a slightly flexed position, whereas the forearm was positioned in a pronated position (Figure 2A). A custom-made, two-point tactile stimulator (Takei; Niigata, Japan) that can control stimulus conditions at a given value using a computer was used to randomly present a total of 10 stimuli to the right index finger pad, which is the contralateral side of the stimulated hemisphere, including one point $(0 \mathrm{~mm}$ between the stimulus pins) or two points (nine stimuli ranging from 1 to $5.0 \mathrm{~mm}$ at $0.5-\mathrm{mm}$ intervals; Figure 2B). We explained
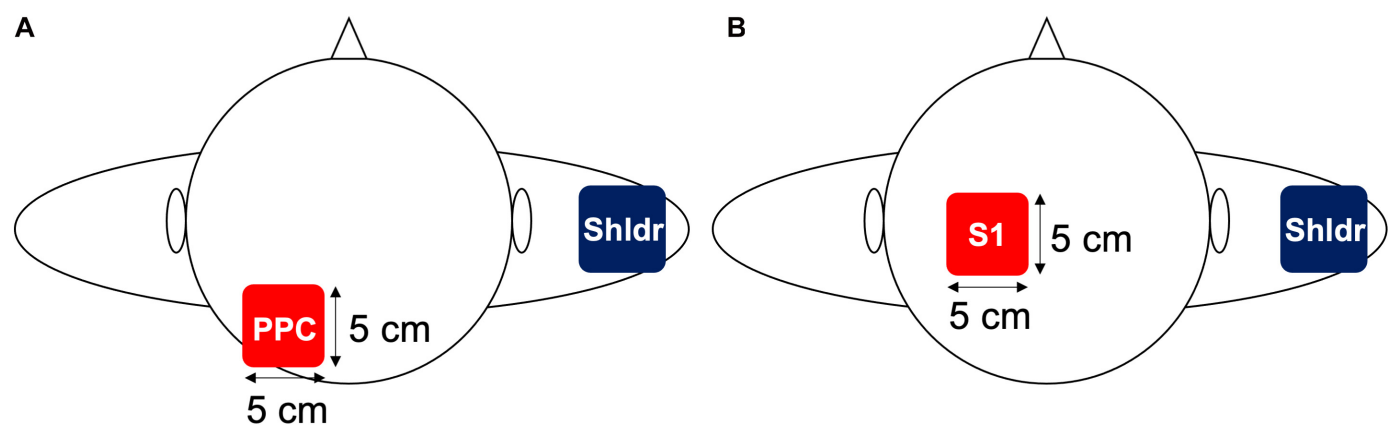

FIGURE 1 | Electrode placement in Experiments 1 and 2. (A) In Experiment 1, an electrode was placed over the left PPC, which is located at P3 in the international 10-20 system, whereas the reference electrode was placed over the contralateral shoulder. (B) In Experiment 2, an electrode was placed over the left S1, which is located $2 \mathrm{~cm}$ posterior to the C3 in the international 10-20 system, whereas the reference electrode was placed over the contralateral shoulder. 

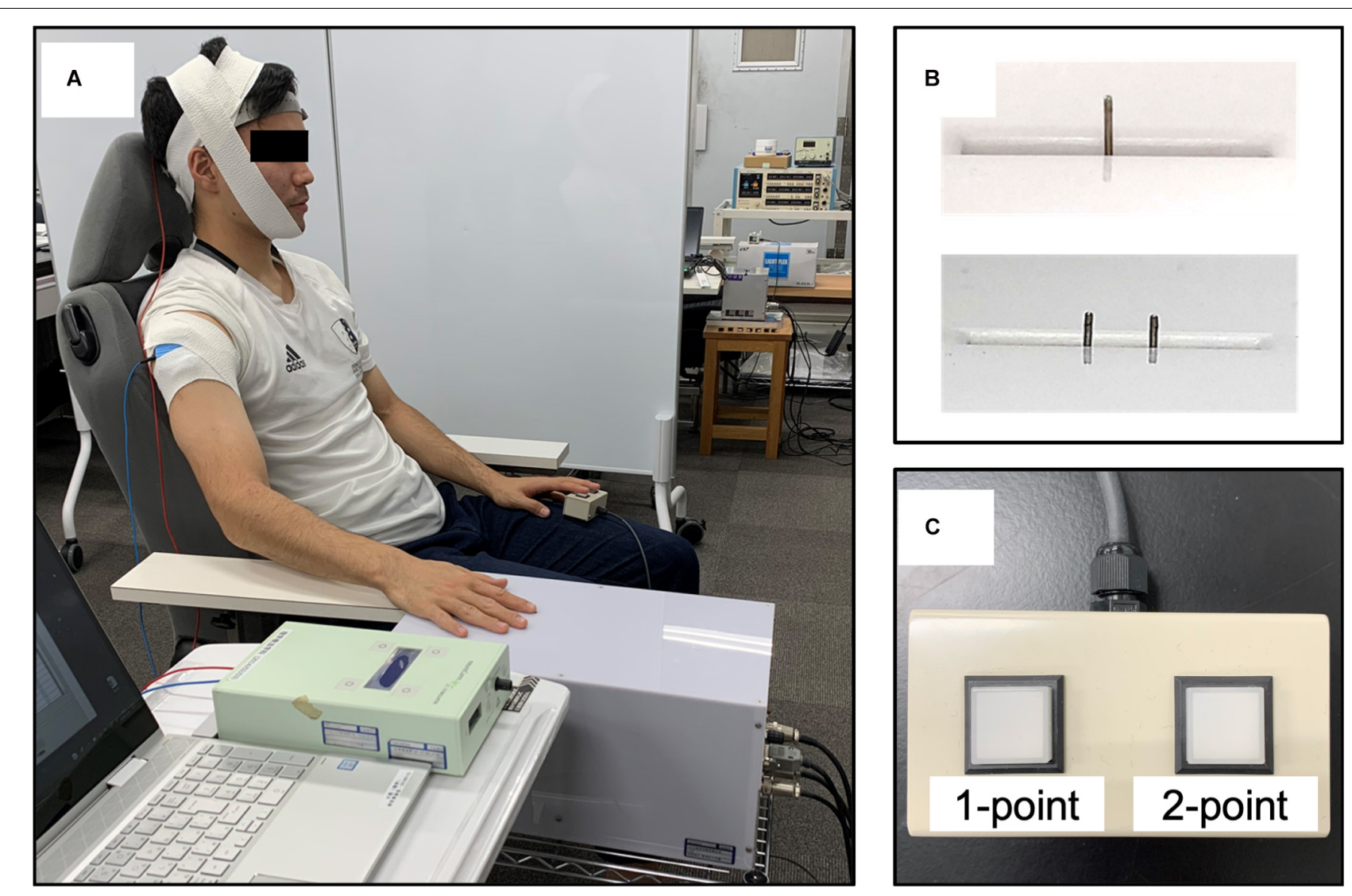

FIGURE 2 | Participants' position and the two-point tactile stimulation device. (A) The participants were seated on a fixed chair with a backrest, with the shoulder and elbow joints slightly flexed and the forearm in a pronated position. (B) The stimulus conditions (stimulus speed, 10.0 mm/s; stimulus penetration depth, 1.0 mm; stimulus presentation time, $1.0 \mathrm{~s}$; and stimulus interval, $5 \mathrm{~s}$ ) were fixed, and pin spacing was randomly assigned (one point, 0 mm and two points, nine different places, ranging from 1.0 to $5.0 \mathrm{~mm}$ at $0.5-\mathrm{mm}$ intervals) using a custom-made computer-controlled two-point tactile stimulation device. (C) Answer button: participants were asked to respond about whether they perceived two points only when they were confident that there were two points, and an uncertain stimulus or one point was classified as one point.

the instructions to the participants that two points should only be considered if they could be clearly identified as two points, whereas other vague stimuli and one point should be defined as one point. The participants were instructed to respond as quickly as possible when they recognized the stimulus by pushing a button held by the left hand. The stimuli were controlled by a computer under the following conditions: stimulus speed, $10.0 \mathrm{~mm} / \mathrm{s}$; stimulus penetration depth, $1.0 \mathrm{~mm}$; and stimulus presentation time, $1.0 \mathrm{~s}$, which were the optimal measurement conditions of TPD, as obtained in our previous study (Yokota et al., 2020) (Figure 2C). Moreover, it was reported that the $\alpha$-band activity decreased in the hemisphere opposite to the one in which visual or tactile attention was directed; however, in this study, we attempted to increase the $\alpha$-band activity in the left hemisphere, which perceives information from the right index finger (Van Dijk et al., 2008; Haegens et al., 2010; Jensen and Mazaheri, 2010). Furthermore, TPD threshold reportedly decreased by gazing at the measurement site during the measurement (Kennett et al., 2001; Moseley and Wiech, 2009; Catley et al., 2014b); therefore, we considered that these conditions could affect the measurement results. To minimize these effects, the participants were instructed to relax and look at a fixed point set at the height of each participant's eyes, at a distance of $\sim 1.5 \mathrm{~m}$, at all times during the experiment.

\section{Experimental Procedures}

All participants first participated in Experiment 1 and then participated in Experiment 2 at least 1 week after the completion of Experiment 1. First, the participants performed 10 practice trials to understand how the stimulus would be presented and how they should respond to the stimulus during the TPD task. After the practice period, TPD was measured in four blocks: two blocks for each of the active and sham conditions, in which 10 different stimulus intervals were presented eight times in each block to obtain 160 measurements for each condition. In the tACS condition of Experiments 1 and 2, tACS was initiated $1 \mathrm{~min}$ before TPD measurements, and the TPD task was performed while receiving consistent stimulation during the block and was stopped immediately after the end of TPD measurements (all participants completed TPD measurements within $8 \mathrm{~min}$ ). In the sham condition, stimulation was initiated $1 \mathrm{~min}$ before the onset of the TPD task but was only applied for $20 \mathrm{~s}$ (10 s of fade in 
and $10 \mathrm{~s}$ of fade out) to avoid stimulation of the cortex during the TPD task. The order of the blocks was randomly assigned for each participant and a 5-min break was introduced between blocks to avoid any carry-over effects from the previous block (Figure 3).

\section{Data and Statistical Analyses}

To analyze the data, the distance between the pins and the correct answer rate were plotted on the $X$-axis and $Y$-axis, respectively, following which we fitted a binomial logistic regression model ["glmfit" function on MATLAB (MathWorks Inc.) with "binomial" and "logit" settings] to the data to draw a psychometric function. Thresholds at the correct rates of 25, 50, and $75 \%$ were calculated for each stimulus condition (tACS or sham) based on the psychophysical curve via logistic regression analysis. Moreover, 50\% threshold was defined as TPD threshold and $75 \%$ threshold minus $25 \%$ threshold was defined as just the noticeable difference (JND), indicating discrimination sensitivity, as previously described (Otsuru et al., 2019). Moreover, the differences in 50\% threshold between the tACS and sham conditions were calculated for the PPC and S1 by subtracting the $50 \%$ threshold calculated in the tACS condition from that calculated in the sham condition. The findings were compared to identify the cortical region that effectively improved TPD after $\alpha$-tACS of either the left PPC or left S1.

For statistical analysis, we performed the Shapiro-Wilk test to verify the normal distribution of each stimulus region for 25,50 , and $75 \%$ thresholds, followed by the two-tailed paired $t$-test with correspondence between stimulus conditions (tACS or sham). Moreover, we analyzed discrimination sensitivity using statistical software (SPSS; IBM) in both Experiments 1 and 2. Furthermore, the differences in the 50\% threshold recorded in the left PPC and left S1 were compared using the two-tailed paired $t$-test. To directly compare the possible regions and to demonstrate the reliability of the TPD measures used in our experiments, we used the two-tailed paired $t$-test to compare the means of the $50 \%$ threshold data obtained from the sham conditions in Experiments 1 and 2. The significance level was set at $5 \%$ for all statistical analyses. Data are expressed as mean \pm standard error (SE).

\section{RESULTS}

\section{Experiment 1. Effects of tACS Over the PPC on the TPD Threshold}

Figures 4A,B show the psychophysical curve fitted via logistic regression analysis obtained in Experiment 1 as well as 50\% TPD threshold, which was calculated from the curve. The 50\% TPD threshold (mean \pm SE) was $2.45 \pm 0.78 \mathrm{~mm}$ in the tACS condition and $2.65 \pm 0.74 \mathrm{~mm}$ in the sham condition. Moreover, TPD threshold decreased in $13(76.5 \%)$ of the 17 participants during the $\alpha$-tACS condition compared with that during the sham condition. The two-tailed paired $t$-test between the two groups revealed a significant decrease in the tACS condition compared with the sham condition $\left[t_{(16)}=2.54, p=0.01\right.$, $r=0.54]$ (Figures 4A,B). JND was $0.68 \pm 0.17$ and $0.81 \pm 0.25$ in the tACS and sham conditions, respectively, with no significant differences between them.

\section{Experiment 2. Effects of $\alpha$-tACS Over the S1 on the TPD Threshold}

Figures 4C,D show the psychophysical curve fitted via logistic regression analysis obtained in Experiment 2 as well as 50\% TPD threshold, which was calculated from the curve. The 50\% TPD threshold (mean \pm SE) was $2.69 \pm 0.66 \mathrm{~mm}$ in the tACS condition and $2.67 \pm 0.59 \mathrm{~mm}$ in the sham condition. Although compared with the sham condition, TPD threshold increased in $11(64.7 \%)$ of the 17 participants during $\alpha$-tACS application, there was no significant difference between the conditions, according to the two-tailed paired $t$-test $\left[t_{(16)}=-0.34, p=0.74, r=0.08\right]$ (Figures 4C,D). JND was $0.56 \pm 0.21$ and $0.58 \pm 0.25$ in the tACS

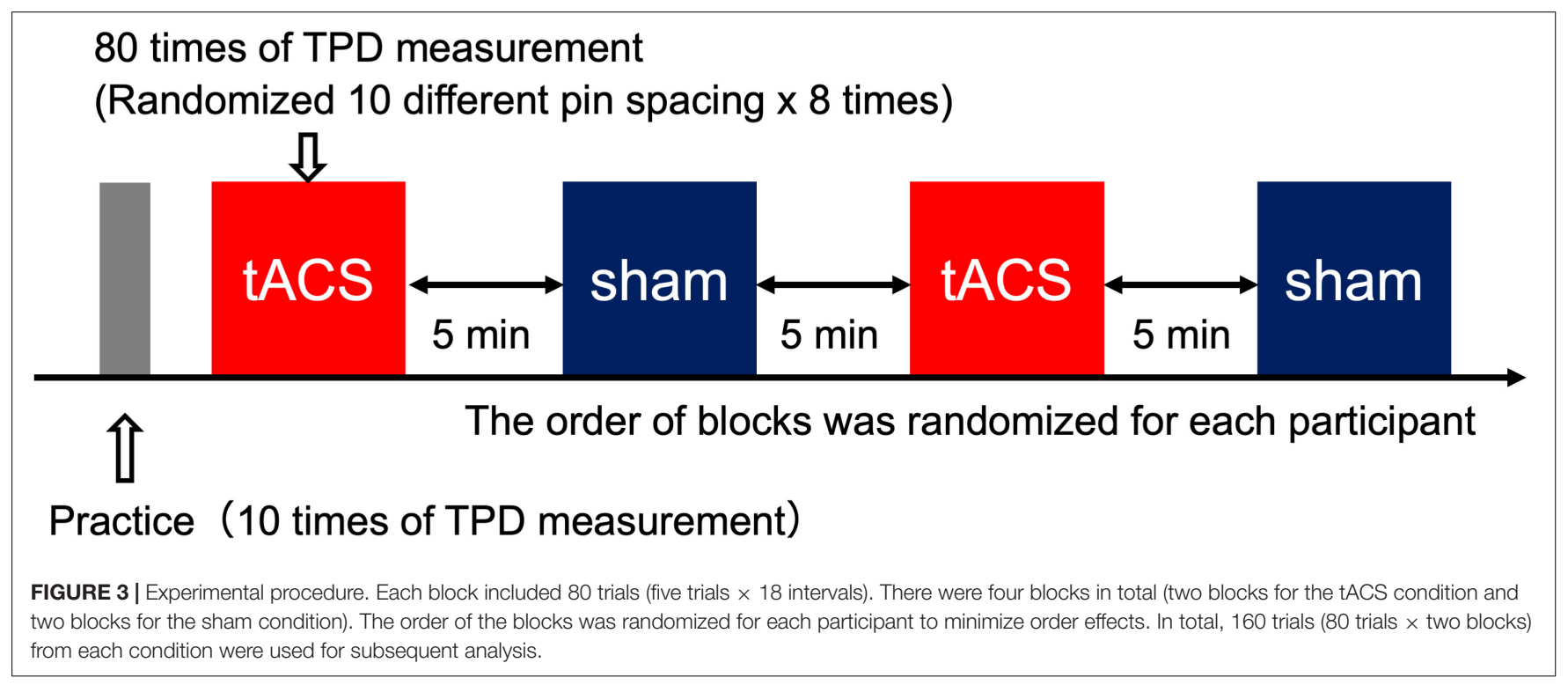




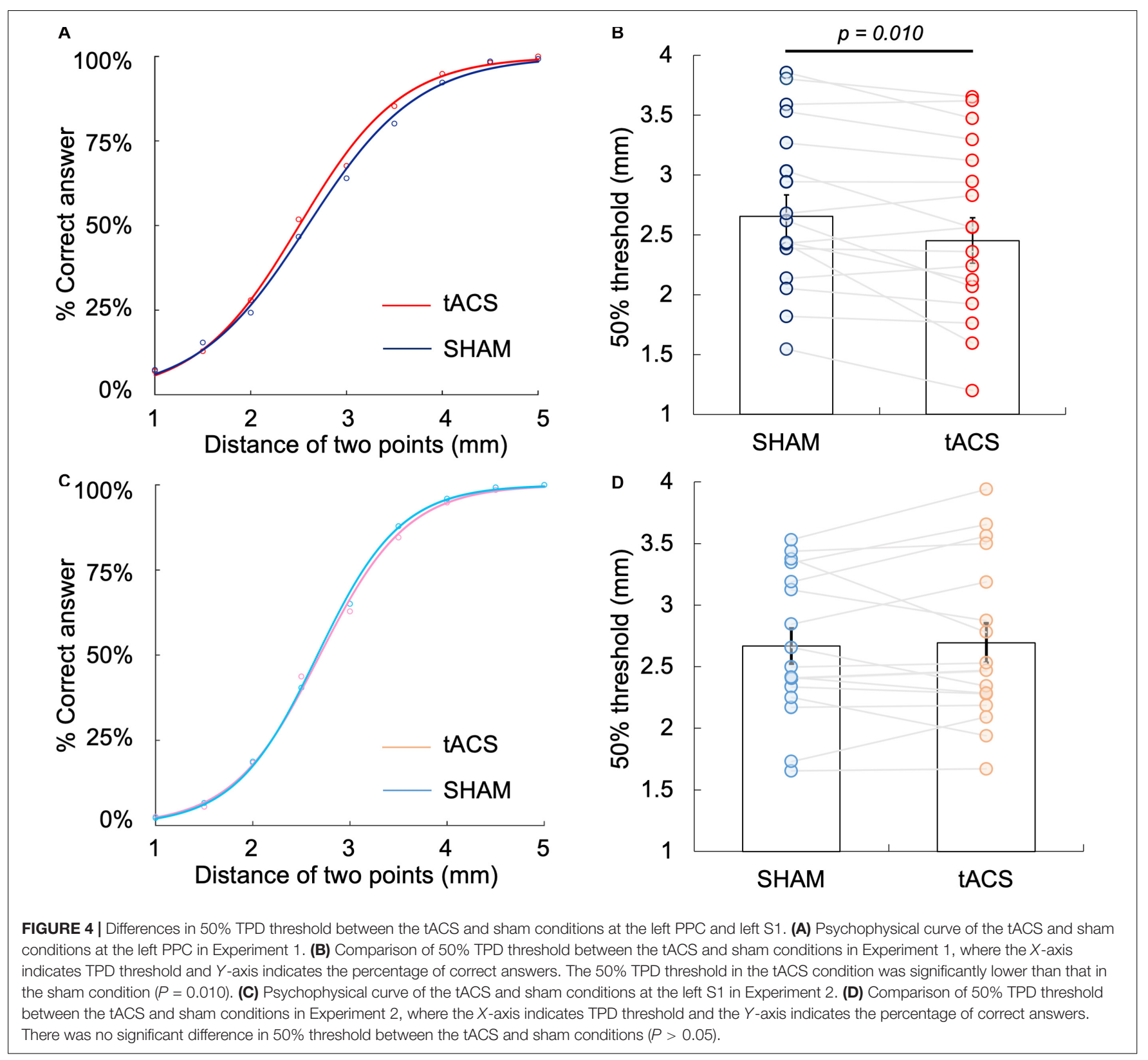

and sham conditions, respectively, with no significant differences between the two conditions.

\section{Comparison of the Differences in $\alpha$-tACS-Induced Threshold Between the Left PPC and Left S1}

Based on the results obtained in Experiments 1 and 2, we compared the differences in $\alpha$-tACS-induced threshold (50\% threshold in the sham condition - 50\% threshold in the tACS condition) in the left PPC and left S1. The differences in $\alpha$-tACSinduced threshold in the left PPC and S1 were $0.2 \pm 0.28$ and $-0.02 \pm 0.27 \mathrm{~mm}$, respectively, with the changes being significantly larger in the left PPC than in the left $\mathrm{S} 1\left[t_{(16)}=3.507\right.$, $p=0.003, r=0.66$, two-tailed] (Figure 5A). However, there was no significant difference in the sham conditions between the regions $\left[t_{(16)}=0.014, p=0.93, r=0.00\right.$, two-tailed] (Figure 5B). Therefore, $\alpha$-tACS over the left PPC effectively lowered the TPD threshold compared with that over the left S1. Moreover, a comparison of the mean of 50\% TPD threshold in sham conditions between Experiments 1 and 2 revealed no statistical difference, which helped ameliorate the reliability of the TPD measures used in our experiments.

\section{DISCUSSION}

The present study aimed to clarify the effects of non-invasive brain stimulation over the left PPC (Experiment 1) and left S1 (Experiment 2), which were suggested to be related to TPD, on 

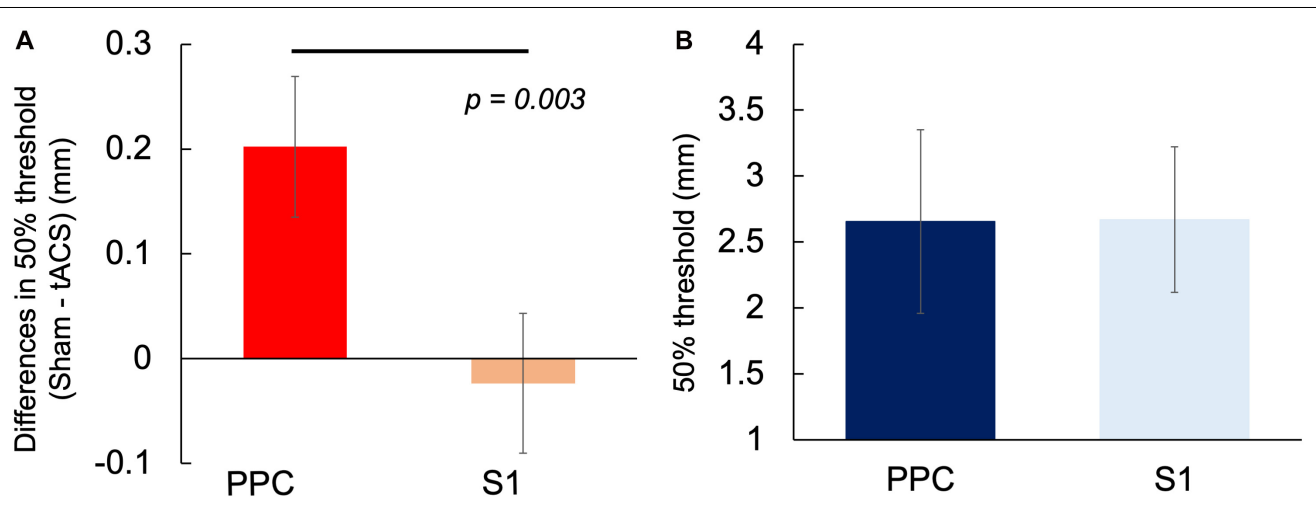

FIGURE 5 | Comparison of the differences in $\alpha$-tACS-induced threshold change and 50\% TPD threshold in the sham condition between the left PPC and left S1. (A) Significant differences in threshold differences (50\% threshold in the sham condition - 50\% threshold in the tACS condition) were observed between the left PPC and the left S1 $(P=0.003)$. (B) No difference was observed in 50\% threshold between the sham condition $(P>0.05)$.

TPD threshold in the right index finger pad using $10-\mathrm{Hz} \alpha$-tACS. The results of Experiment 1 illustrated that $10-\mathrm{Hz} \alpha$-tACS of the left PPC, which is the area that plays a vital role in the TPD process and is responsible for attentional control, decreased TPD threshold. In contrast, in Experiment 2, similar stimulation with $\alpha$-tACS over the left S1, which is suggested to be involved in tactile signal transfer to the higher-order regions of TPD, yielded no changes in TPD threshold. Furthermore, a comparison of the effects of $\alpha$-tACS on the left PPC and left S1 based on the differences in 50\% TPD threshold revealed that $\alpha$-tACS applied over the left PPC significantly decreases TPD threshold compared with that applied over the left S1.

A possible explanation for the improvement of TPD following $10-\mathrm{Hz} \alpha$-tACS over the left PPC is the increased $\alpha$-band activity caused by $\alpha$-tACS. Previous studies have shown that $\alpha$-tACS enhances $\alpha$-band activity at the stimulated region (Zaehle et al., 2010; Helfrich et al., 2014; Vossen et al., 2015; Berger et al., 2018); therefore, $\alpha$-band activity at the left PPC might have increased under the tACS condition in the present study; this suggests that this increase in $\alpha$-band activity improves TPD. This result is consistent with that of previous studies related to the effect of $\alpha$-band activity on performance during cognitive tasks (Dockree et al., 2007; Braboszcz and Delorme, 2011). Dockree et al. (2007) reported that individuals who performed well on a workingmemory task requiring sustained attention for an average of 24 blocks of 4.7 min exhibited a significant increase in $\alpha$-band activity in the occipitoparietal region during the task. Moreover, in tasks requiring sustained attention for a long period, such as a $28 \mathrm{~min} \times 2$ session auditory discrimination task and a $20 \mathrm{~min} \times 3$ block respiratory-rate counting task, the $\alpha$ power decreased when participants provided incorrect answers during the task on the simultaneously measured EEG in the same individuals (Makeig and Inlow, 1993; Dockree et al., 2007; Braboszcz and Delorme, 2011). Furthermore, in a temporal order judgment task using electrical stimulation to the left and right index fingers, a 10$\mathrm{Hz} \alpha$-tACS applied over the PPC, similar to the present study, reportedly improved the discrimination ability, suggesting the involvement of an increased $\alpha$-band activity in the PPC in the cognitive task (Otsuru et al., 2019). As the TPD task used in this study is a cognitive task requiring prolonged and sustained attention (for approximately $10 \mathrm{~min}$ in four blocks, with a 5min rest period between blocks), the $\alpha$-tACS-induced increase in $\alpha$-band activity in the left PPC might have promoted sustained attention to the task and improved task performance. In other words, because $\alpha$-band activity is considered to exert a gating effect that suppresses signal transduction by increasing its power (Jensen and Mazaheri, 2010; Foxe and Snyder, 2011; Jensen et al., 2014; Van Diepen et al., 2019), increased $\alpha$-band activity can be deemed to inhibit the left PPC and prevent switching attention away from the task to distractors (Yantis et al., 2002).

Unlike Experiment 1, $\alpha$-tACS of the left S1 in Experiment 2 did not yield any change in TPD. Similar to visual attention, previous studies have shown that when an electrical stimulus is administered to the index finger, the $\alpha$-band activity in the S1 on the opposite side of the stimulus decreases (Haegens et al., 2010; Gould et al., 2011). This is important because $\gamma$-band activity in the high-frequency region $(30-150 \mathrm{~Hz})$, which is important for information transmission, is periodically suppressed by $\alpha$-band activity in a phase-dependent manner. This is referred to as cross-frequency phase-amplitude coupling (Klimesch et al., 2007; Osipova et al., 2008; Haegens et al., 2011b; Roux et al., 2013; Jensen et al., 2014). Therefore, in the region involved in the task, it is considered that information transfer is facilitated by a burst of $\gamma$-band activity that occurs during the trough of $\alpha$-band activity (Jensen and Mazaheri, 2010; Jensen et al., 2014; Bonnefond and Jensen, 2015; Gips et al., 2016). Moreover, increased $\alpha$-band activity in the S1 is reportedly correlated with the decreased frequency of neural firing in macaque monkeys and decreased excitability in human MEG and fMRI studies (Osipova et al., 2008; Haegens et al., 2011b; Scheeringa et al., 2011). These data suggest that $\alpha$-band activity in the $S 1$, which is a relay point of haptic information, needs to decrease physiologically to increase neural firing, thereby facilitating information transfer and improving TPD. In contrast, if the $\alpha$-band activity in the S1 was decreased during the application of $\alpha$-tACS, similar to the previously reported decrease in $\alpha$-band activity after $\alpha$-tACS application (Gundlach et al., 2017), it may facilitate information transfer and decrease TPD threshold. However, in this study, 
no significant difference in TPD threshold was observed when $\alpha$-tACS was applied over the S1. Therefore, our results did not support our initial hypothesis that $\alpha$-tACS increases $\alpha$-band activity during stimulation, similar to the previous findings in the visual and parietal cortexes (Helfrich et al., 2014; Vossen et al., 2015; Berger et al., 2018), and thus, disturbed information transfer. That is, the $\alpha$-tACS over the S1 used in this experiment may not have an effect on $\alpha$-band activity. However, recent studies have suggested that discrimination sensitivity is not affected even though $\alpha$-band activity increases in the visual domain (Samaha et al., 2017; Iemi and Busch, 2018; Samaha et al., 2020). Therefore, further studies are warranted to clarify the causal relationship between $\alpha$-tACS and $\alpha$-band activity in the S1.

Furthermore, on comparing the differences in TPD threshold because of $\alpha$-tACS $(50 \%$ threshold in the sham condition $50 \%$ threshold in the tACS condition) applied over the left PPC and left S1 in Experiments 1 and 2, respectively, the amount of change in the left PPC was significantly larger than that in the left S1. Akatsuka et al. (2008) demonstrated that the inferior parietal lobule in the left PPC selectively showed significantly higher activity during the TPD task in a study that compared cortical activity during the TPD task with the task of discriminating the intensity of stimuli using fMRI. Because increases in $\alpha$-band activity are critical for cognitive tasks requiring sustained attention (Makeig and Inlow, 1993; Dockree et al., 2007; Braboszcz and Delorme, 2011; Otsuru et al., 2019) and considering that the PPC plays a pivotal role in attentional control (Corbetta et al., 2000; Yantis et al., 2002), our result supports previous results that the left PPC plays an important role in the TPD process (Akatsuka et al., 2008). Moreover, the S1 is important in the process of information processing of TPD and decreased $\alpha$-band activity must be further decreased to increase neural activity in the S1 such that it can facilitate signal transfer to higher-order regions, such as the PPC. Although this appeared to be paradoxical, the hierarchical mechanism of increases and decreases in $\alpha$-band activities is controlled by a top-down regulation (Sadaghiani and Kleinschmidt, 2016; Van Diepen et al., 2019); this appears to be crucial for the optimal function of TPD. Therefore, although we did not measure the actual $\alpha$-band activity during $\alpha$-tACS application and the focal increment in $\alpha$-band activity in each cortical region was not supported, it might have been separately modulated by $\alpha$-tACS to some extent in our experiment, as proposed in previous studies (Zaehle et al., 2010; Helfrich et al., 2014; Vossen et al., 2015; Berger et al., 2018); it might have affected TPD processing, thereby differently shifting TPD threshold depending on the role of $\alpha$-band activity in each region in the TPD process.

The reliability of the TPD measurement method was a major issue in the present study, as previous studies questioned the reliability of TPD as a measure of perceptual function (Catley et al., 2013; Tong et al., 2013). Slowly adapting I fiber and rapidly adapting I fiber are the primary afferents involved in TPD (Johansson and Vallbo, 1979; Darian-Smith and Kenins, 1980; Craig and Lyle, 2001). Moreover, the frequency of firing of these afferents, including their receptors, changes according to stimulus intensity (Vega-Bermudez and Johnson, 1999a,b). Therefore, the manner in which the stimulus pins are applied during measurements may affect the test results. To overcome this limitation, we used a custom-made twopoint tactile stimulator that can control the stimulus speed and stimulus penetration depth of the stimulation pins at the time of measurement to arbitrary values on the computer, thereby providing a comprehensive measurement of TPD threshold. Accordingly, we reported that the threshold value was the lowest at a stimulus speed of $10.0 \mathrm{~mm} / \mathrm{s}$ and a stimulus penetration depth of $1.0 \mathrm{~mm}$, which represents a reliable measurement method for studying the effects of these interventions (Yokota et al., 2020). There was no significant difference in the mean $50 \%$ threshold in the sham conditions between Experiments 1 and 2. This indicates that the TPD measurement method used in this experiment was indeed reliable. Therefore, the TPDs used in this experiment clearly demonstrate the region-specific effects of $\alpha$-tACS on the left PPC and left S1 because they may accurately reflect the changes in higher-order perceptual functions caused by $\alpha$-tACS. In the future, the potential clinical applications of these results, such as whether similar results can be obtained in patients with impaired TPD, should be explored.

This study had some limitations in its experimental method. Previous studies have reported changes in cortical oscillatory activity induced by $\alpha$-tACS (Zaehle et al., 2010; Helfrich et al., 2014; Berger et al., 2018); therefore, we attempted to increase $\alpha$-band activity in the left PPC and S1 by administering 10$\mathrm{Hz} \alpha$-tACS. However, we did not record the actual brain oscillatory activity in the cortical region. Therefore, another recently reported mechanism of $\alpha$-tACS-induced improvement of the TPD, i.e., the entraining of the cortical neurons by the rhythmic activity from the skin's peripheral nerves under the electrode input via the sensory pathway (Asamoah et al., 2019), should be considered. It is also reported that in rats and cadavers, high-intensity currents exceeding $4 \mathrm{~mA}$ are needed to directly phase-entrain brain rhythms (Vöröslakos et al., 2018). In this manner, the entrainment of the cortical activity caused by $\alpha$-tACS in the present study might not only be focal under the electrode because the rhythmic activity was transmitted via the sensory pathway. Moreover, we have not measured actual brain activity during $\alpha$-tACS or simulated electrical field distribution; therefore, it is not possible to explain exactly how $\alpha$-tACS affects cortical oscillatory activity in the left PPC and left S1. Furthermore, the placement of the reference electrode should be considered. Although we placed the reference electrode over the shoulder contralateral to the stimulating electrode to minimize the occurrence of phosphenes, according to previous studies (Mehta et al., 2015; Miyaguchi et al., 2018), the placement of the distant electrode reportedly impacts not only the area under the electrode but also the surrounding area (Faria et al., 2011; Karabanov et al., 2019). The extracephalic reference also increases the stimulation effects on subcortical brain regions (Mehta et al., 2015). Furthermore, the current flow and electrical field distribution by the $\alpha$-tACS montage used in this experiment were not simulated; therefore, the effects of $\alpha$-tACS might not be less focal than expected. Therefore, elucidating whether the effects of $\alpha$-tACS were focal to the left PPC and impossible on the left S1 and that there may be some overlap between the S1 and PPC is essential. However, $\alpha$-tACS was found to exert 
different effects over the left PPC and left S1; therefore, the specific reactions of each region could be demonstrated to some extent. Detailed studies, such as those using a device that can simultaneously measure brain oscillatory activity during $\alpha$-tACS and perform a simulation of the electrical field distribution, are warranted to confirm these results in the future.

\section{CONCLUSION}

The present study reveals that the use of $10-\mathrm{Hz} \alpha$-tACS over the left PPC effectively reduces TPD threshold; however, a similar stimulation over the left $\mathrm{S} 1$ does not yield any significant changes. The effect of $10-\mathrm{Hz} \alpha$-tACS on the determination of TPD threshold was region-specific in each cortical region involved in TPD. However, our results are speculative because actual cortical activity was not measured; therefore, additional studies are warranted to confirm the causal relationship between $\alpha$-tACS in the left PPC and sustained attention or $\alpha$-tACS in the S1, and information processing during the determination of TPD threshold using a more precise experimental paradigm, such as the concurrent measurement of cortical activity, during $10-\mathrm{Hz}$ $\alpha$-tACS.

\section{DATA AVAILABILITY STATEMENT}

The raw data supporting the conclusions of this article will be made available by the authors, without undue reservation.

\section{REFERENCES}

Akatsuka, K., Noguchi, Y., Harada, T., Sadato, N., and Kakigi, R. (2008). Neural codes for somatosensory two-point discrimination in inferior parietal lobule: an fMRI study. Neuroimage 40, 852-858. doi: 10.1016/j.neuroimage.2007. 12.013

Akatsuka, K., Wasaka, T., Nakata, H., Kida, T., Hoshiyama, M., Tamura, Y., et al. (2007). Objective examination for two-point stimulation using a somatosensory oddball paradigm: an MEG study. Clin. Neurophysiol. 118, 403-411. doi: 10. 1016/j.clinph.2006.09.030

Antal, A., Alekseichuk, I., Bikson, M., Brockmoller, J., Brunoni, A. R., Chen, R., et al. (2017). Low intensity transcranial electric stimulation: safety, ethical, legal regulatory and application guidelines. Clin. Neurophysiol. 128, 1774-1809. doi: 10.1016/j.clinph.2017.06.001

Asamoah, B., Khatoun, A., and Mc Laughlin, M. (2019). tACS motor system effects can be caused by transcutaneous stimulation of peripheral nerves. Nat. Commun. 10, 266.

Bassetti, C., Bogousslavsky, J., and Regli, F. (1993). Sensory syndromes in parietal stroke. Neurology 43, 1942-1949. doi: 10.1212/wnl.43.10.1942

Baumgarten, T. J., Schnitzler, A., and Lange, J. (2016). Prestimulus alpha power influences tactile temporal perceptual discrimination and confidence in decisions. Cereb. Cortex 26, 891-903. doi: 10.1093/cercor/bhu247

Berger, A., Pixa, N. H., Steinberg, F., and Doppelmayr, M. (2018). Brain oscillatory and hemodynamic activity in a bimanual coordination task following transcranial alternating current stimulation (tACS): a combined EEGfNIRS study. Front. Behav. Neurosci. 12:67. doi: 10.3389/fnbeh.2018.00067

Bonnefond, M., and Jensen, O. (2015). Gamma activity coupled to alpha phase as a mechanism for top-down controlled gating. PLoS One 10:e0128667. doi: 10.1371/journal.pone.0128667

Braboszcz, C., and Delorme, A. (2011). Lost in thoughts: neural markers of low alertness during mind wandering. Neuroimage 54, 3040-3047. doi: 10.1016/j. neuroimage.2010.10.008

\section{ETHICS STATEMENT}

The studies involving human participants were reviewed and approved by the Niigata University of Health and Welfare. The patients/participants provided their written informed consent to participate in this study.

\section{AUTHOR CONTRIBUTIONS}

$\mathrm{HY}$ and $\mathrm{HO}$ conceived the study and designed the experiments. KS and SK performed the experiments. NO interpreted the data. SM, YI, and KN helped draft the manuscript. All authors approved the final version of the submitted manuscript.

\section{FUNDING}

This work was supported by a Grant-in-Aid for Young Scientists 20K19429 and a Grant-in-Aid for Scientific Research (A) 19H01090 from the Japan Society for the Promotion of Science.

\section{ACKNOWLEDGMENTS}

The authors would like to thank Enago (www.enago.jp) for the English language review.

Catley, M. J., O'connell, N. E., Berryman, C., Ayhan, F. F., and Moseley, G. L. (2014a). Is tactile acuity altered in people with chronic pain? a systematic review and meta-analysis. J. Pain 15, 985-1000. doi: 10.1016/j.jpain.2014.06.009

Catley, M. J., Tabor, A., Miegel, R. G., Wand, B. M., Spence, C., and Moseley, G. L. (2014b). Show me the skin! Does seeing the back enhance tactile acuity at the back? Man Ther. 19, 461-466. doi: 10.1016/j.math.2014.04.015

Catley, M. J., Tabor, A., Wand, B. M., and Moseley, G. L. (2013). Assessing tactile acuity in rheumatology and musculoskeletal medicine-how reliable are twopoint discrimination tests at the neck, hand, back and foot? Rheumatology (Oxford) 52, 1454-1461. doi: 10.1093/rheumatology/ket140

Clayton, M. S., Yeung, N., and Cohen Kadosh, R. (2018). The many characters of visual alpha oscillations. Eur. J. Neurosci. 48, 2498-2508. doi: 10.1111/ejn.13747

Corbetta, M., Kincade, J. M., Ollinger, J. M., Mcavoy, M. P., and Shulman, G. L. (2000). Voluntary orienting is dissociated from target detection in human posterior parietal cortex. Nat. Neurosci. 3, 292-297. doi: 10.1038/73009

Craddock, M., Poliakoff, E., El-Deredy, W., Klepousniotou, E., and Lloyd, D. M. (2017). Pre-stimulus alpha oscillations over somatosensory cortex predict tactile misperceptions. Neuropsychologia 96, 9-18. doi: 10.1016/j. neuropsychologia.2016.12.030

Craig, J. C., and Lyle, K. B. (2001). A comparison of tactile spatial sensitivity on the palm and fingerpad. Percept. Psychophys. 63, 337-347. doi: 10.3758/bf03194474

Darian-Smith, I., and Kenins, P. (1980). Innervation density of mechanoreceptive fibres supplying glabrous skin of the monkey's index finger. J. Physiol. 309, 147-155. doi: 10.1113/jphysiol.1980.sp013500

Dinse, H. R., Kleibel, N., Kalisch, T., Ragert, P., Wilimzig, C., and Tegenthoff, M. (2006). Tactile coactivation resets age-related decline of human tactile discrimination. Ann. Neurol. 60, 88-94. doi: 10.1002/ana.20862

Dockree, P. M., Kelly, S. P., Foxe, J. J., Reilly, R. B., and Robertson, I. H. (2007). Optimal sustained attention is linked to the spectral content of background EEG activity: greater ongoing tonic alpha (approximately $10 \mathrm{~Hz}$ ) power supports successful phasic goal activation. Eur. J. Neurosci. 25, 900-907. doi: 10.1111/ j.1460-9568.2007.05324.x 
Faria, P., Hallett, M., and Miranda, P. C. (2011). A finite element analysis of the effect of electrode area and inter-electrode distance on the spatial distribution of the current density in tDCS. J. Neural Eng. 8, 066017. doi: 10.1088/17412560/8/6/066017

Foxe, J. J., and Snyder, A. C. (2011). The role of alpha-band brain oscillations as a sensory suppression mechanism during selective attention. Front. Psychol. 2:154. doi: 10.3389/fpsyg.2011.00154

Gips, B., Van Der Eerden, J. P., and Jensen, O. (2016). A biologically plausible mechanism for neuronal coding organized by the phase of alpha oscillations. Eur. J. Neurosci. 44, 2147-2161. doi: 10.1111/ejn.13318

Godde, B., Stauffenberg, B., Spengler, F., and Dinse, H. R. (2000). Tactile coactivation-induced changes in spatial discrimination performance. J. Neurosci. 20, 1597-1604. doi: 10.1523/jneurosci.20-04-0 1597.2000

Gould, I. C., Rushworth, M. F., and Nobre, A. C. (2011). Indexing the graded allocation of visuospatial attention using anticipatory alpha oscillations. J. Neurophysiol. 105, 1318-1326. doi: 10.1152/jn.00653.2010

Gundlach, C., Muller, M. M., Nierhaus, T., Villringer, A., and Sehm, B. (2017). Modulation of somatosensory alpha rhythm by transcranial alternating current stimulation at mu-frequency. Front. Hum. Neurosci. 11:432. doi: 10.3389/ fnhum.2017.00432

Haegens, S., Händel, B. F., and Jensen, O. (2011a). Top-down controlled alpha band activity in somatosensory areas determines behavioral performance in a discrimination task. J. Neurosci. 31, 5197-5204. doi: 10.1523/jneurosci.519910.2011

Haegens, S., Nacher, V., Luna, R., Romo, R., and Jensen, O. (2011b). AlphaOscillations in the monkey sensorimotor network influence discrimination performance by rhythmical inhibition of neuronal spiking. Proc. Natl. Acad. Sci. U.S.A. 108, 19377-19382. doi: 10.1073/pnas.1117190108

Haegens, S., Osipova, D., Oostenveld, R., and Jensen, O. (2010). Somatosensory working memory performance in humans depends on both engagement and disengagement of regions in a distributed network. Hum. Brain Mapp. 31, 26-35.

Halgren, M., Ulbert, I., Bastuji, H., Fabó, D., Erõss, L., Rey, M., et al. (2019). The generation and propagation of the human alpha rhythm. Proc. Natl. Acad. Sci. U.S.A. 116, 23772-23782.

Harvie, D. S., Edmond-Hank, G., and Smith, A. D. (2018). Tactile acuity is reduced in people with chronic neck pain. Musculoskelet. Sci. Pract. 33, 61-66. doi: 10.1016/j.msksp.2017.11.009

Helfrich, R. F., Schneider, T. R., Rach, S., Trautmann-Lengsfeld, S. A., Engel, A. K., and Herrmann, C. S. (2014). Entrainment of brain oscillations by transcranial alternating current stimulation. Curr. Biol. 24, 333-339. doi: 10.1016/j.cub. 2013.12.041

Heriseanu, R., Baguley, I. J., and Slewa-Younan, S. (2005). Two-point discrimination following traumatic brain injury. J. Clin. Neurosci. 12, 156-160. doi: 10.1016/j.jocn.2004.03.029

Iemi, L., and Busch, N. A. (2018). Moment-to-moment fluctuations in neuronal excitability bias subjective perception rather than strategic decision-making. eNeuro 5:ENEURO.0430-17.2018. doi: 10.1523/ENEURO.0430-17.2018

Iemi, L., Chaumon, M., Crouzet, S. M., and Busch, N. A. (2017). Spontaneous neural oscillations bias perception by modulating baseline excitability. J. Neurosci. 37, 807-819. doi: 10.1523/jneurosci.1432-16.2016

Im, C. H., Park, J. H., Shim, M., Chang, W. H., and Kim, Y. H. (2012). Evaluation of local electric fields generated by transcranial direct current stimulation with an extracephalic reference electrode based on realistic 3D body modeling. Phys. Med. Biol. 57, 2137-2150. doi: 10.1088/0031-9155/57/8/2137

Inui, K., Wang, X., Tamura, Y., Kaneoke, Y., and Kakigi, R. (2004). Serial processing in the human somatosensory system. Cereb. Cortex 14, 851-857. doi: 10.1093/ cercor/bhh043

Jensen, O., Gips, B., Bergmann, T. O., and Bonnefond, M. (2014). Temporal coding organized by coupled alpha and gamma oscillations prioritize visual processing. Trends Neurosci. 37, 357-369. doi: 10.1016/j.tins.2014.04.001

Jensen, O., and Mazaheri, A. (2010). Shaping functional architecture by oscillatory alpha activity: gating by inhibition. Front Hum Neurosci 4:186. doi: 10.3389/ fnhum.2010.00186

Johansson, R. S., and Vallbo, A. B. (1979). Tactile sensibility in the human hand: relative and absolute densities of four types of mechanoreceptive units in glabrous skin. J. Physiol. 286, 283-300. doi: 10.1113/jphysiol.1979.sp012619
Jones, S. R., Kerr, C. E., Wan, Q., Pritchett, D. L., Hämäläinen, M., and Moore, C. I. (2010). Cued spatial attention drives functionally relevant modulation of the mu rhythm in primary somatosensory cortex. J. Neurosci. 30, 13760-13765. doi: 10.1523/jneurosci.2969-10.2010

Jones, S. R., Pritchett, D. L., Sikora, M. A., Stufflebeam, S. M., Hämäläinen, M., and Moore, C. I. (2009). Quantitative analysis and biophysically realistic neural modeling of the MEG mu rhythm: rhythmogenesis and modulation of sensoryevoked responses. J. Neurophysiol. 102, 3554-3572. doi: 10.1152/jn.00535. 2009

Karabanov, A. N., Saturnino, G. B., Thielscher, A., and Siebner, H. R. (2019). Can transcranial electrical stimulation localize brain function? Front. Psychol. 10:213. doi: 10.3389/fpsyg.2019.00213

Kennett, S., Taylor-Clarke, M., and Haggard, P. (2001). Noninformative vision improves the spatial resolution of touch in humans. Curr. Biol. 11, 1188-1191. doi: 10.1016/s0960-9822(01)00327-x

Klimesch, W., Sauseng, P., and Hanslmayr, S. (2007). EEG alpha oscillations: the inhibition-timing hypothesis. Brain Res. Rev. 53, 63-88. doi: 10.1016/j. brainresrev.2006.06.003

Lange, J., Halacz, J., Van Dijk, H., Kahlbrock, N., and Schnitzler, A. (2012). Fluctuations of prestimulus oscillatory power predict subjective perception of tactile simultaneity. Cereb. Cortex 22, 2564-2574. doi: 10.1093/cercor/bhr329

Makeig, S., and Inlow, M. (1993). Lapses in alertness: coherence of fluctuations in performance and EEG spectrum. Electroencephalogr. Clin. Neurophysiol. 86, 23-35. doi: 10.1016/0013-4694(93)90064-3

Mehta, A. R., Pogosyan, A., Brown, P., and Brittain, J. S. (2015). Montage matters: the influence of transcranial alternating current stimulation on human physiological tremor. Brain Stimul. 8, 260-268. doi: 10.1016/j.brs.2014.11.003

Miyaguchi, S., Otsuru, N., Kojima, S., Saito, K., Inukai, Y., Masaki, M., et al. (2018). Transcranial alternating current stimulation with gamma oscillations over the primary motor cortex and cerebellar hemisphere improved visuomotor performance. Front. Behav. Neurosci. 12:132. doi: 10.3389/fnbeh.2018.00132

Moseley, G. L., and Wiech, K. (2009). The effect of tactile discrimination training is enhanced when patients watch the reflected image of their unaffected limb during training. Pain 144, 314-319. doi: 10.1016/j.pain.2009.04.030

Osipova, D., Hermes, D., and Jensen, O. (2008). Gamma power is phase-locked to posterior alpha activity. PLoS One 3:e3990. doi: 10.1371/journal.pone.0003990

Otsuru, N., Kamijo, K., Otsuki, T., Kojima, S., Miyaguchi, S., Saito, K., et al. (2019). $10 \mathrm{~Hz}$ transcranial alternating current stimulation over posterior parietal cortex facilitates tactile temporal order judgment. Behav. Brain Res. 368:111899. doi: 10.1016/j.bbr.2019.111899

Pleger, B., Foerster, A. F., Ragert, P., Dinse, H. R., Schwenkreis, P., Malin, J. P., et al. (2003). Functional imaging of perceptual learning in human primary and secondary somatosensory cortex. Neuron 40, 643-653. doi: 10.1016/s08966273(03)00677-9

Pleger, B., Wilimzig, C., Nicolas, V., Kalisch, T., Ragert, P., Tegenthoff, M., et al. (2016). A complementary role of intracortical inhibition in age-related tactile degradation and its remodelling in humans. Sci. Rep. 6:27388.

Raco, V., Bauer, R., Olenik, M., Brkic, D., and Gharabaghi, A. (2014). Neurosensory effects of transcranial alternating current stimulation. Brain Stimul. 7, 823-831. doi: 10.1016/j.brs.2014.08.005

Ragert, P., Dinse, H. R., Pleger, B., Wilimzig, C., Frombach, E., Schwenkreis, P., et al. (2003). Combination of $5 \mathrm{~Hz}$ repetitive transcranial magnetic stimulation (rTMS) and tactile coactivation boosts tactile discrimination in humans. Neurosci. Lett. 348, 105-108. doi: 10.1016/s0304-3940(03)00745-6

Ragert, P., Kalisch, T., Bliem, B., Franzkowiak, S., and Dinse, H. R. (2008). Differential effects of tactile high- and low-frequency stimulation on tactile discrimination in human subjects. BMC Neurosci. 9:9. doi: 10.1186/1471-22029-9

Roux, F., Wibral, M., Singer, W., Aru, J., and Uhlhaas, P. J. (2013). The phase of thalamic alpha activity modulates cortical gamma-band activity: evidence from resting-state MEG recordings. J. Neurosci. 33, 17827-17835. doi: 10.1523/ jneurosci.5778-12.2013

Sadaghiani, S., and Kleinschmidt, A. (2016). Brain networks and alpha-oscillations: structural and functional foundations of cognitive control. Trends Cogn. Sci. 20, 805-817. doi: 10.1016/j.tics.2016.09.004

Samaha, J., Iemi, L., Haegens, S., and Busch, N. A. (2020). Spontaneous brain oscillations and perceptual decision-making. Trends Cogn. Sci. 24, 639-653. doi: 10.1016/j.tics.2020.05.004 
Samaha, J., Iemi, L., and Postle, B. R. (2017). Prestimulus alpha-band power biases visual discrimination confidence, but not accuracy. Conscious Cogn. 54, 47-55. doi: 10.1016/j.concog.2017.02.005

Scheeringa, R., Mazaheri, A., Bojak, I., Norris, D. G., and Kleinschmidt, A. (2011). Modulation of visually evoked cortical FMRI responses by phase of ongoing occipital alpha oscillations. J. Neurosci. 31, 3813-3820. doi: 10.1523/jneurosci. 4697-10.2011

Schmidt-Wilcke, T., Wulms, N., Heba, S., Pleger, B., Puts, N. A., Glaubitz, B., et al. (2018). Structural changes in brain morphology induced by brief periods of repetitive sensory stimulation. Neuroimage 165, 148-157. doi: 10.1016/j. neuroimage.2017.10.016

Shahbabaie, A., Hatami, J., Farhoudian, A., Ekhtiari, H., Khatibi, A., and Nitsche, M. A. (2018). Optimizing electrode montages of transcranial direct current stimulation for attentional bias modification in early abstinent methamphetamine users. Front. Pharmacol. 9:907. doi: 10.3389/fphar.2018. 00907

Sliva, D. D., Black, C. J., Bowary, P., Agrawal, U., Santoyo, J. F., Philip, N. S., et al. (2018). A prospective study of the impact of transcranial alternating current stimulation on EEG correlates of somatosensory perception. Front. Psychol. 9:2117. doi: 10.3389/fpsyg.2018.02117

Sripati, A. P., Yoshioka, T., Denchev, P., Hsiao, S. S., and Johnson, K. O. (2006). Spatiotemporal receptive fields of peripheral afferents and cortical area $3 \mathrm{~b}$ and 1 neurons in the primate somatosensory system. J. Neurosci. 26, 2101-2114. doi: 10.1523/jneurosci.3720-05.2006

Stevens, J. C., and Cruz, L. A. (1996). Spatial acuity of touch: ubiquitous decline with aging revealed by repeated threshold testing. Somatosens. Mot. Res. 13, 1-10. doi: 10.3109/08990229609028907

Tegenthoff, M., Ragert, P., Pleger, B., Schwenkreis, P., Forster, A. F., Nicolas, V., et al. (2005). Improvement of tactile discrimination performance and enlargement of cortical somatosensory maps after 5 Hz rTMS. PLoS Biol. 3:e362. doi: 10.1371/journal.pbio.0030362

Thut, G., Nietzel, A., Brandt, S. A., and Pascual-Leone, A. (2006). Alpha-band electroencephalographic activity over occipital cortex indexes visuospatial attention bias and predicts visual target detection. J. Neurosci. 26, 9494-9502. doi: 10.1523/jneurosci.0875-06.2006

Tong, J., Mao, O., and Goldreich, D. (2013). Two-point orientation discrimination versus the traditional two-point test for tactile spatial acuity assessment. Front. Hum. Neurosci. 7:579. doi: 10.3389/fnhum.2013.00579

Van Diepen, R. M., Foxe, J. J., and Mazaheri, A. (2019). The functional role of alphaband activity in attentional processing: the current zeitgeist and future outlook. Curr. Opin. Psychol. 29, 229-238. doi: 10.1016/j.copsyc.2019.03.015
Van Dijk, H., Schoffelen, J. M., Oostenveld, R., and Jensen, O. (2008). Prestimulus oscillatory activity in the alpha band predicts visual discrimination ability. J. Neurosci. 28, 1816-1823. doi: 10.1523/jneurosci.1853-07.2008

Vega-Bermudez, F., and Johnson, K. O. (1999a). SA1 and RA receptive fields, response variability, and population responses mapped with a probe array. J. Neurophysiol. 81, 2701-2710. doi: 10.1152/jn.1999.81.6.2701

Vega-Bermudez, F., and Johnson, K. O. (1999b). Surround suppression in the responses of primate SAl and RA mechanoreceptive afferents mapped with a probe array. J. Neurophysiol. 81, 2711-2719. doi: 10.1152/jn.1999.81.6.2711

Vöröslakos, M., Takeuchi, Y., Brinyiczki, K., Zombori, T., Oliva, A., FernándezRuiz, A., et al. (2018). Direct effects of transcranial electric stimulation on brain circuits in rats and humans. Nat. Commun. 9:483.

Vossen, A., Gross, J., and Thut, G. (2015). Alpha power increase after transcranial alternating current stimulation at alpha frequency (alpha-tACS) reflects plastic changes rather than entrainment. Brain Stimul. 8, 499-508. doi: 10.1016/j.brs. 2014.12.004

Yantis, S., Schwarzbach, J., Serences, J. T., Carlson, R. L., Steinmetz, M. A., Pekar, J. J., et al. (2002). Transient neural activity in human parietal cortex during spatial attention shifts. Nat. Neurosci. 5, 995-1002. doi: 10.1038/nn921

Yokota, H., Otsuru, N., Kikuchi, R., Suzuki, R., Kojima, S., Saito, K., et al. (2020). Establishment of optimal two-point discrimination test method and consideration of reproducibility. Neurosci. Lett. 714:134525. doi: 10.1016/j. neulet.2019.134525

Zaehle, T., Rach, S., and Herrmann, C. S. (2010). Transcranial alternating current stimulation enhances individual alpha activity in human EEG. PLoS One 5:e13766. doi: 10.1371/journal.pone.0013766

Zhang, Y., and Ding, M. (2010). Detection of a weak somatosensory stimulus: role of the prestimulus mu rhythm and its top-down modulation. J. Cogn. Neurosci. 22, 307-322. doi: 10.1162/jocn.2009.21247

Conflict of Interest: The authors declare that the research was conducted in the absence of any commercial or financial relationships that could be construed as a potential conflict of interest.

Copyright (c) 2021 Yokota, Otsuru, Saito, Kojima, Miyaguchi, Inukai, Nagasaka and Onishi. This is an open-access article distributed under the terms of the Creative Commons Attribution License (CC BY). The use, distribution or reproduction in other forums is permitted, provided the original author(s) and the copyright owner(s) are credited and that the original publication in this journal is cited, in accordance with accepted academic practice. No use, distribution or reproduction is permitted which does not comply with these terms. 